\title{
Basic research and model systems in familial dysautonomia: What do we know and what's next?
}

\author{
Antonio Heras-Garvin ${ }^{1}$
}

Received: 29 June 2017/ Accepted: 1 July 2017/Published online: 13 July 2017

(C) Springer-Verlag GmbH Germany 2017

Familial dysautonomia (FD), also known as Riley-Day syndrome or hereditary sensory autonomic neuropathy type III, is a rare autosomal recessive disease affecting almost exclusively the Ashkenazi Jewish population, with an incidence of 1:3700 live births and a carrier frequency ranging from one in 19 (in those of Polish descent) to one in 32. FD patients manifest from birth a broad range of symptoms, including vomiting crises, difficulties in swallowing, gastrointestinal and cardiovascular dysfunction, ataxia, pneumonia, kyphoscoliosis, absence of fungiform papillae on the tongue, lack of baseline tears, difficulties in regulating body temperature and blood pressure, and reduced sensitivity to pain and temperature. Although symptomatic treatments are available [6], there is no therapy that can slow, halt, or revert the progression of the disease, and patients have significant morbidity and mortality [5].

FD is caused by mutations in the IKBKAP gene, which encodes the I $\kappa \mathrm{B}$ kinase complex-associated protein (IKAP; also known as ELP1), a component of the transcriptional elongator complex. The most common mutation in the IKBKAP gene is a transition from $\mathrm{T}$ to $\mathrm{C}$ at position 6 of the $5^{\prime}$ splice site of $I K B K A P$ intron 20 . This mutation alters the splicing pattern of the IKBKAP gene specifically in nervous system tissues, leading to a decreased synthesis of the IKAP/hELP1 protein $[1,7]$. The reduction of IKAP/ hELP1 protein levels has an effect on the development, function, and survival of various neural crest-derived lineages, such as afferent autonomic neurons and sensory

Antonio Heras-Garvin

antonio.heras@i-med.ac.at

1 Department of Neurology, Medical University of Innsbruck, Innsbruck, Austria neurons, but the molecular mechanisms underlying these neural crest tissue-specific defects are still unclear.

Imaging and autopsy data indicate that compared to healthy controls FD patients have white matter abnormalities, optic neuropathy, and abnormal development with progressive degeneration and depletion of sensory and autonomic neurons. Specifically, the lack of IKAP results in the size and cell numbers of dorsal-root ganglion and superior cervical ganglion in FD patients being severely reduced. Taken together, these pieces of information indicate that FD is the result of developmental defects plus a neurodegenerative component [2, 5].

In this issue of Clinical Autonomic Research, Lefcort and colleagues review the in vivo and in vitro modelsused in the past and being used nowadays - to clarify and reveal the molecular and cellular mechanisms underlying and mediating the pathophysiology of FD [3]. By using animal and in vitro models, several basic research groups have contributed importantly in the last years to a better understanding of the functions of IKAP/hELP1 and its possible role on this devastating neuropathy. Among all these research groups, the group by Lefcort and colleagues at Montana State University focus their work on the generation of mouse models of FD by deleting the Ikbkap gene from different neuronal populations. Their results, as well as the data obtained by other groups using further model systems, are described in detail by the authors in this issue.

The review by Lefcort describes the different in vivo and in vitro models of FD that have been developed so far and the relevant findings obtained using these models. Interestingly, the authors clarify how different non-mammalian and mouse models have been crucial to elucidate the specific roles of IKAP and their relation with the clinical symptoms and disease progression. And in spite of the fact that the function of IKAP/hELP1 has not yet been 
totally elucidated, these cellular and animal models have been key to discover that IKAP/hELP1 is involved in transcription elongation, $\alpha$-tubulin acetylation, intracellular trafficking, exocytosis, and tRNA post-transcriptional modifications. It has also been shown that IKAP/hELP1 plays an essential role in the development of the nervous system and, importantly, in neuronal cell survival, migration and differentiation $[4,5]$.

The authors also discuss the interesting and important recent findings obtained through the use of induced pluripotential stem cells obtained from FD patients, which constitutes a powerful in vitro model to study the molecular mechanism underlying this fatal disease and a potential tool for drug screening. Lefcort and colleagues are judicious when summarizing the strengths and weaknesses of the different FD models to approach unanswered questions. Finally, the authors discuss the potential therapeutic strategies to target neurodegeneration in FD, all of which have emerged from the model systems in the last years [3].

The alliance between clinical and basic research and, especially, the use of model systems has been essential to clarify many aspects of FD. However, although our molecular, cellular and pathological understanding of this disease has increased in recent years, new models and approaches are required to completely define its causes and, hopefully in a not too distant future, to find a cure.

\section{Compliance with ethical standards}

Conflict of interests None.

\section{References}

1. Anderson SL, Coli R, Daly IW, Kichula EA, Rork MJ, Volpi SA, Ekstein J, Rubin BY (2001) Familial dysautonomia is caused by mutations of the IKAP gene. Am J Hum Genet 68:753-758

2. Dietrich P, Dragatsis I (2016) Familial dysautonomia: mechanisms and models. Genet Mol Biol 39:497-514

3. Lefcort F, Mergy M, Ohlen SB, Ueki Y, George L (2017) Animal and cellular models of familial dysautonomia. Clin Auton Res. doi: 10.1007/s10286-017-0438-2

4. Nguyen L, Humbert S, Saudou F, Chariot A (2010) Elongator-an emerging role in neurological disorders. Trends Mol Med 16:1-6

5. Norcliffe-Kaufmann L, Slaugenhaupt SA, Kaufmann H (2017) Familial dysautonomia: history, genotype, phenotype and translational research. Prog Neurobiol 152:131-148

6. Palma JA, Norcliffe-Kaufmann L, Fuente-Mora C, Percival L, Mendoza-Santiesteban C, Kaufmann H (2014) Current treatments in familial dysautonomia. Expert Opin Pharmacother 15:2653-2671

7. Slaugenhaupt SA, Blumenfeld A, Gill SP, Leyne M, Mull J, Cuajungco MP, Liebert CB, Chadwick B, Idelson M, Reznik L, Robbins C, Makalowska I, Brownstein M, Krappmann D, Scheidereit C, Maayan C, Axelrod FB, Gusella JF (2001) Tissue-specific expression of a splicing mutation in the IKBKAP gene causes Familial dysautonomia. Am J Hum Genet 68:598-605 